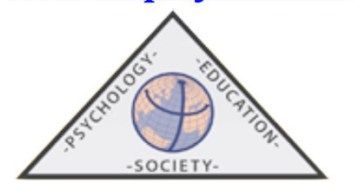

\title{
La figura de asesoría en procesos en la implementación del aprendizaje basado en proyectos en el aula
}

\author{
Maria del Carmen DIAZ GARCIA ${ }^{1}$ y Teresa GARCÍA GÓMEZ ${ }^{2}$ \\ ${ }^{(1)}$ Centro Profesorado de Almería \\ (2)Universidad de Almería
}

(Recibido el 13 de Junio de 2019, Aceptado el 15 de Mayo de 2020)

RESUMEN: La asesoría en procesos o asesoría colaborativa, como figura de acompañamiento y facilitador de procesos de formación que recalan en la práctica docente ha sido el principal objeto del estudio del presente trabajo. En concreto, se ha tratado de analizar de qué manera está figura influye en la transformación del proceso de enseñanza-aprendizaje de una docente cuando decide incorporar el Aprendizaje Basado en Proyectos (ABP) en su práctica de aula. Se ha realizado a través de una investigación de corte cualitativo, concretamente un estudio de caso colectivo de base instrumental, cuyos objetivos han sido: analizar la transformación que produce la puesta en práctica del ABP en las creencias educativas del profesorado; reflejar cómo experimentan los docentes la transformación del proceso de enseñanza en la puesta en marcha del ABP; estudiar la influencia del ABP en el proceso de aprendizaje del alumnado; analizar cómo el asesoramiento en procesos incide en los cambios de procesos de enseñanza y aprendizaje; $y$ ver la influencia del centro educativo en las prácticas educativas a través del Aprendizaje Basado en Proyectos. Los resultados de uno de los casos constatan que la figura de la asesoría en procesos o colaborativa incide de manera positiva en la transformación de la práctica educativa, siendo crucial para la innovación educativa.

Palabras clave: asesoramiento colaborativo; formación permanente; innovación educativa; metodología cualitativa; estudio de caso.

\section{The Concept of Process Advice in the Implementation of Project-Based Learning in the Classroom}

\begin{abstract}
The main object of study in this work has been the advice in process or collaborative advice, as a means of accompaniment and facilitator for training processes that fit into teaching practice. Specifically, the aim has been to analyse how this concept affects the transformation of the teaching-learning process of a teacher when deciding to include the Project-Based Learning (PBL) in his or her classroom practice. This work has been carried out through a qualitative research, specifically an instrumental-based collective case study, whose objectives have been: to analyse the transformation that the implementation of PBL brings about in the educational beliefs of teachers; to reflect how teachers experience the transformation of the teaching process in the implementation of PBL; to study the influence of PBL on the student learning process; to analyse how the advice in processes has an impact on changes in teaching and learning processes; and to assess the
\end{abstract}


influence of the school on the educational practices through Project-Based Learning. From the results of one of the cases, it can be seen that the concept of advice in processes or collaborative advice has a positive impact on the transformation of the educational practice, thus being crucial for the innovative education.

Keywords: Collaborative Advice; Permanent Education; Educational Innovation; Qualitative Methodology; Case Study.

Correspondencia: María del Carmen Díaz García. Paseo de la Caridad, 125, 04008, Almería. E-mail: mcarmen.diaz.garcia.edu@juntadeandalucia.es

\section{La formación permanente del profesorado}

En la Comunidad Autónoma de Andalucía, la formación permanente del profesorado empieza articularse con la creación de los centros de profesorado con la Orden de 20 de mayo de 1992. En esta Orden se establece el I Plan Andaluz de Formación del Profesorado, donde se recogen las Líneas Estratégicas de Formación Permanente, y aparece por primera vez la figura de asesoría de formación. En esta época comienza a introducirse en torno a la formación términos de investigación en acción, un nuevo concepto de currículo, los proyectos, la triangulación y la reflexión. Se inicia de este modo una ilusión al cambio, hacia caminos más progresistas.

Tras el análisis de los posteriores Planes Andaluces de Formación del Profesorado, en concreto el II en 2003 y III Plan en 2013, se puede observar un distanciamiento de un modelo de formación basado en un enfoque individualista en sus inicios hacia una visión más centrada en formación en los centros. Es especialmente en el III Plan Andaluz de Formación (Decreto 93/2013) donde se establecen unas líneas estratégicas que inciden en la mejora y aplicación de metodologías innovadoras en las aulas, relacionándolas desde un marcado enfoque competencial. Desde estas líneas estratégicas, la función de los centros del profesorado se ha encaminado a realizar una oferta formativa intentado influir en las prácticas educativas. Pero, tal y como señalan Mckinsey \& Company (2012):

En el ámbito de la formación, España tiene un amplio recorrido de mejora. Es cierto que comparados con el resto de países de la OCDE, en España contamos con un número de horas de formación suficiente. El problema es que la efectividad de la formación no depende tanto del número de horas, como del contenido de las mismas. (p. 12)

Fullan (2002) señala que el profesorado cambia sus creencias y actitudes cuando ve que la formación que recibe repercute en el aprendizaje del alumnado de manera significativa, por lo que el análisis de la formación ofrecida por los centros del profesorado y, más concretamente, del papel desempeñado por la asesoría de referencia en los centros educativos y con los docentes puede estar estrechamente relacionado con el calado de la formación en su práctica educativa en el aula.

Es en este contexto, donde nos planteamos conocer y comprender la incidencia de la asesoría de referencia, como figura de acompañamiento y facilitador de procesos de formación que recalan en la práctica.

\subsection{El asesoramiento en procesos}

Con las tareas encomendadas a los docentes del siglo XXI, como señala Imbernon (2014), éstos se han convertido en profesionales de la educación y del conocimiento debiendo adaptarse 
a los avances científicos-tecnológicos y sociales que tan deprisa se están viviendo en la sociedad actualmente.

Esto nos hace replantearnos nuevos enfoques y cambios ante las formas de enseñar y aprender en la escuela y, por tanto, dejar aparcados métodos utilizados en la enseñanza tradicional. Imbernon (2017) escribe que "el profesorado sabe que ha de estar en constante cambio y renovación y que la incertidumbre forma parte desde hace tiempo de su entorno profesional" (p. 22). Las funciones en las aulas y en las instituciones educativas han ido cambiando a lo largo de los siglos al mismo ritmo que la sociedad; ahora el sistema educativo debe acelerar si no quiere perder el paso y quedarse obsoleto.

Pero a veces estos cambios y renovaciones que debe afrontar el profesorado en su docencia son llevados a cabo de manera individual, sin apenas cuestionarse el porqué de los mismos y si de verdad son adecuados a las circunstancias y al contexto en el que se encuentran. Esto nos marca el replanteamiento de las nuevas modalidades de asesoramientos alejándonos de modelos donde, como señala Zeichner (1995),

Se desarrolla la visión de los profesores como técnicos, cuya función consiste en la mera aplicación de lo que otros, ausentes de las aulas, quieren que hagan y en la aceptación de modo de reforma educativa de arriba abajo en donde los profesores sólo aparecen como participantes pasivos (p.75).

Es aquí donde entraría el papel fundamental de la asesoría de referencia y el replanteamiento de un cambio de su rol tradicional.

El asesor hasta ahora concebido como un gestor de formación, como una fuente de recursos y como un simple administrativo sometido a la burocracia (modelo de asesoramiento tradicional) tiene que avanzar hacia una concepción más centrada en el equipo docente, en el centro y en los procesos de mejora como vía de desarrollo profesional (modelo de asesoramiento en proceso) (Pinya y Rosselló, 2014, p. 663).

Acompañar a los docentes hacia un desarrollo profesional desde el replanteamiento de políticas (gestión del centro), prácticas (en lo que se enseña y cómo se enseña) y culturas (relaciones, valores y creencias educativas) instauradas en el centro (Booth y Ainscow, 2015) es tarea de la asesoría colaborativa y desde la que este estudio se ha centrado para guiar cambios en los procesos que están tan anclados en el quehacer docente. Es decir, en este proceso de investigación se ha ofrecido un acompañamiento por parte de la asesoría a una docente que ha querido transformar su práctica de aula, en donde la asesoría en concreto ha asumido la responsabilidad de guiar los cambios e interpretar las demandas en función de las prácticas que se han ido desarrollando en el aula. Como señala Ventura (2008), entendiendo el asesoramiento como "una forma de reconocer las dificultades y adversidades cuando la tarea pedagógica supone cambiar todo o parte de aquello que está establecido para mejorar la escuela” (p. 2). También se ha analizado de qué manera influye el aprendizaje basado en proyectos en el cambio de creencias docentes y en el aprendizaje del alumnado.

\subsection{Aprendizaje Basado en Proyectos}

El Método de Proyectos (Kilpatrick, 1918; Sainz, 1934; Hernández, 1996) o más actualmente Aprendizaje Basado en Proyectos (ABP) (Trujillo, 2012; Vergara, 2015) supone un cambio en las maneras de hacer dominantes en la escuela. Implica promover espacios en los que el 
alumnado se cuestione, participe, adquiera estrategias para la resolución de sus preguntas estableciendo conexiones entre las diferentes áreas de conocimiento. Un espacio en el que se vive una participación democrática y donde la gestión del aula es compartida y enriquecida por todas las personas que la componen.

Todo esto supone un cambio en las creencias del profesorado sobre su acción educativa, tomando conciencia que si de lo que se trata es de formar a ciudadanos competentes para el siglo XXI, la tarea docente consistirá en plantear situaciones en las cuales el alumnado pueda construir, modificar y reformular conocimientos, actitudes, habilidades, emociones y valores (Vergara, 2015); así como crear experiencias relevantes, cultivar la cultura de la producción y retroalimentar, reflexionar y revisar en el aula (Grossman, Dean, Kavanagh y Herrmann, 2019).

Es por ello que el cambio que se pide al profesorado con la formación es complejo, ya que requiere de tiempo, de una base sólida que deje poco espacio a la incertidumbre, una adaptación al contexto en el que se desarrolla, y precisa de un periodo experiencial en el que el cambio se lleve a cabo y se vaya incorporando en las propias vivencias profesionales (Imbernon, 2007). Si no queremos que "la creatividad presente en el origen pasa a ser reemplazada por una versión técnica de la metodología, que se reduce a una serie de pasos que hay que dar para que el ABP funcione con eficacia" (Torrego y Méndez, 2018, p. 4), cobra especial importancia de la figura de la asesoría en procesos para poder acompañar en estos tiempos (Jacobsen, 2019), en este llevar a la práctica aquello en lo que se forman y poder compartir esos periodos de incertidumbre en los que se necesita de un apoyo cuando los pasos hacia un nuevo camino se entornan inestables.

\section{Objetivos}

Los objetivos planteados en esta investigación han sido los siguientes:

a. Analizar la transformación que produce la puesta en práctica del Aprendizaje Basado en Proyectos en las creencias educativas del profesorado.

b. Reflejar cómo experimentan los docentes la transformación del proceso de enseñanza en la puesta en marcha del Aprendizaje Basado en Proyectos en sus aulas.

c. Estudiar la influencia de la puesta en práctica del Aprendizaje Basado en Proyectos en el proceso de aprendizaje del alumnado.

d. Analizar como el asesoramiento en procesos incide en los cambios de procesos de enseñanza y aprendizaje.

e. Ver la influencia del centro educativo en las prácticas educativas a través del Aprendizaje Basado en Proyectos.

\section{Metodología}

El estudio que presentamos es de corte cualitativo, concretamente es una investigación realizada con estudio de caso, para analizar en profundidad un procedimiento, en particular el proceso de cambio de las prácticas de dos docentes en la experimentación del ABP en sus aulas, acompañadas por la figura de la asesoría colaborativa o en proceso, así como los cambios que se producen en los procesos de aprendizaje del alumnado. Este estudio de caso es de base instrumental, más concretamente es un estudio de caso colectivo (Stake, 1998), ya que vamos a elegir 
dos casos de entre los posibles para comprender la influencia y el cambio que se genera por el asesoramiento colaborativo en el ABP en su puesta en práctica y los cambios en el proceso de aprendizaje del alumnado, en dos centros diferentes. Cada uno será el instrumento para comprender nuestro objeto de estudio.

A la hora de seleccionar los casos hemos tenido en cuenta "el tipo de estudio de caso que se desea realizar, dónde está ubicado el caso, qué es lo que va a generar mayor comprensión, los costes de desplazamiento y el tiempo que va a emplear" (Simons, 2011, p. 53). En definitiva, hemos maximizado el criterio, como señalan Vázquez y Angulo (2003, p. 19), de "elegir el caso que ofrezca las mejores y mayores oportunidades de aprendizaje, con el que podamos aprender en profundidad la problemática seleccionada".

Teniendo en cuenta esto, los criterios que guiaron la selección de los casos a estudiar fueron los siguientes:

- Ubicación del centro: centro en la provincia de Almería con facilidad de acceso.

- Estabilidad docente: profesorado con destino definitivo en el centro.

- Historial de formación: profesorado con formación en aprendizaje basado en proyectos.

En concreto, este trabajo se basa en el estudio del primer caso, realizado con una docente de un centro de educación infantil y primaria de la provincia de Almería, que comienza a dar sus primeros pasos en la puesta en práctica del aprendizaje basado en proyectos, a raíz de una actividad formativa llevada a cabo en su centro ofertada por el Centro de Profesorado de Almería, y en el que es acompañada por la asesoría de referencia.

\subsection{Instrumentos de recogida de datos y análisis.}

Dentro de la variedad existente de instrumentos de recogida de datos, determinamos como los más adecuados a utilizar para lograr nuestros objetivos: la observación participante, la entrevista semiestructurada en profundidad y el análisis de documentos.

En cuanto a la observación participante, hemos tenido la oportunidad de meternos de lleno en el campo, observando a los sujetos investigados (profesorado y alumnado) desde una perspectiva de un miembro que también influye en lo que se observa debido a su participación (Flick, 2004). La observación participante se ha realizado durante seis meses.

Las entrevistas semiestructuradas en profundidad nos han ayudado a captar lo que la persona investigada piensa, cree e interpreta del mundo que le rodea, en concreto su acción en el aula y su proceso de transformación, complementando a las observaciones realizadas (Stake, 1998; Woods, 1987). En concreto hemos realizado 1 entrevista a la maestra y 3 entrevistas grupales al alumnado.

Por último, "el estudio de documentos sigue el mismo esquema de razonamiento que la observación o la entrevista (...) Hay que valorar con antelación la posible utilidad de los diferentes documentos y distribuir el tiempo de modo que se emplee razonablemente" (Stake, 1998, p.66). En el marco de esta investigación el análisis del diario de aula de la docente (desde enero hasta junio) nos ha ayudado a recoger aquellas impresiones y emociones que ha ido experimentando la maestra durante la puesta en practica del aprendizaje basado en proyectos, así como con el proceso de acompañamiento de la asesoría de referencia. También el análisis de la programación 
de aula nos ha ayudado ir vislumbrado de qué manera ha ido la docente introduciendo cambios en su práctica de aula y alejándose de la totalidad de prácticas basadas en el libro de texto.

Abandonamos la recogida de datos cuando llegamos a la saturación teórica y el análisis de los mismos comenzó desde el principio de la recogida de información para ir dotando de sentido las primeras impresiones (Stake, 1998). Se realizó la triangulación entre los diferentes instrumentos de recogida de datos y entre las distintas fuentes de información como procedimiento para obtener validez y fiabilidad de los datos, contrastando estos permanentemente y evitar así toda posible distorsión.

El análisis inductivo de los datos se ha realizado mediante el método comparativo constante, descomponiéndolos en segmentos, codificándolos para poder clasificarlos y encontrar conexiones entre ellos, patrones, conceptos, y poder verificarlos, identificando sus propiedades, estableciendo sus interrelaciones e integrándolos en una teoría coherente que explique el caso (Simons, 2011). Es decir, hemos realizado un análisis de contenido por medio de una codificación teórica abierta que nos permitiera el desarrollo de una teoría fundamentada, que generara inductivamente ideas teóricas a partir de los datos, puesto que en ningún momento teníamos la intención de comprobar una teoría ya existente.

\section{Resultados}

El análisis realizado nos ha permitido empezar a vislumbrar todo el entresijo de relaciones y actuaciones que se llevan a cabo dentro de los inicios de puesta en práctica del ABP. Examinando la transformación que produce la puesta en práctica del ABP en las creencias educativas del profesorado, hemos podido comprobar como esta docente ha ido cambiando la percepción con respecto al aprendizaje del alumnado, desde un enfoque más tradicional, considerando éste como una práctica individual hacia una concepción del aprendizaje más autónomo. Va asumiendo un papel de guía, ayudando al alumnado cuando lo necesita y dejando espacios para que éste vaya tomando decisiones respeto a la organización, al contenido y a la calidad de su trabajo

Mi función como docente ha sido más bien de guía. No les he ayudado en casi nada, más bien les he guiado en lo que ellos querían hacer... (Entrevista maestra).

Aprender y trabajar implica silencio, reflejo de la concepción individualista que tiene la maestra del aprendizaje, algo que demanda constantemente en los inicios del proyecto. Sin embargo, conforme se va avanzando en este, va tolerando cierto nivel de ruido, considerándolo como propio de un ambiente de aprendizaje grupal a la vista del progreso que el alumnado va realizando en su propio proceso de aprendizaje

Es cierto que a mi siempre me ha molestado mucho el ruido, y he intentado siempre controlar todo lo que en mi aula sucedía, precisamente para evitar alborotos y estas cosas. Pero he aprendido a ceder un poco en este sentido (y te aseguro que era algo impensable hace poco), y la verdad es que he salido beneficiada. (Reflexión final de la maestra)

Otro cambio en la práctica de la maestra es dar tiempo para aprendizaje de nuevas adquisiciones, tanto en lo que se refiere al alumnado como a ella. La docente ha comenzado a ser consciente de los aprendizajes realizados y de sus lagunas; comienza a percibir la importancia de plantearse el para qué, el sentido de la innovación metodológica, más allá de la motivación 
del alumnado, para lo que es necesario un conocimiento previo de la metodología que se va a emplear antes de iniciarla en el aula.

Durante el proceso de realización del proyecto en el aula la docente ha experimentado diferentes emociones que han ido reflejando la transformación de su proceso de enseñanza. Afloran miedos en relación a dar autonomía al alumnado en su aprendizaje, basados en la idea de que algunos grupos no van a trabajar, reflejando que el aprendizaje tiene lugar cuando la enseñanza es directiva. En definitiva, miedos por el desconocimiento de cómo llevar a cabo la gestión del aula, dónde poner la frontera de la no intervención o hasta donde intervenir durante el proceso para evitar ser directiva

Bueno, eso me pasó sobre todo al principio, que no sabía lo que tenía que hacer, hasta donde me podía meter yo en el grupo, hasta dónde podía yo orientarlos, todo eso al principio no lo sabía. Por eso fue quizás a lo mejor lo que más me agobió, pero bueno cuando ya se termina ya ves hasta donde te puedes meter, que los puedes orientar. Es cierto que algunos grupos están más perdidos que otros, pues al que esté más perdido ya se le puede orientar un poquito más... pero al principio no sabía hasta que punto yo podía implicarme con ellos... y bueno, creo que aún sigo sin saberlo (se ríe) Imagino que sale sólo. La necesidad de cada uno pues te van pidiendo lo que necesitan. (Entrevista maestra)

El proceso vivido por la docente refleja un recorrido de dependencia de la asesoría a una mayor autonomía, en el que ella se ve influenciada por el feedback del alumnado, que se encuentra motivado en el trabajo que está realizando. Feedback que está en relación, como reflejan las palabras de la docente, en que el alumnado realice lo que de él se espera, no tanto una reflexión de lo que esta metodología le posibilita en sus aprendizajes frente a una metodología tradicional, transmisiva, que denomina de lápiz y papel.

El resumen de esta sesión para mí, es que los niños estuvieron encantados, trabajaron motivados, hicieron todo lo que se les pidió sin rechistar, y estuvieron muy participativos en todo momento. Está claro que trabajar así les gusta mucho más que trabajar de la manera tradicional, es decir, con lápiz y papel. (Reflexión maestra)

En cuanto al alumnado, la influencia del ABP en su proceso de aprendizaje, hemos podido comprobar como este percibe un cambio metodológico en el aula y la mejora en las relaciones y organización de la misma. Durante el proceso de trabajo ha experimentado cambios, ya que ha tenido la posibilidad de autorregulación, trabajo en equipo, asumir responsabilidades, escuchar a sus iguales y ayudar a sus compañeros y compañeras

Hemos aprendido a hacer trabajo cooperativo, estar en grupo ayudando los unos a los otros, a buscar información, a realizar trabajos con mucha ayuda, a organizarnos bien, bueno algunos no mucho pero nos hemos ayudado, a aprender a ser mayores y a madurar un poquito porque con estos trabajos hemos aprendido primero a buscar la información, hemos realizado power point, que ha costado, y que nos lo hemos pasado muy bien. Ha sido como hacer tareas pero hemos hecho así, muy divertido, muy rapidillo. (Entrevista alumnado)

Al mismo tiempo, el ABP ha hecho que el alumnado no vea a la maestra como el centro y eje sobre el que gira el proceso de enseñanza-aprendizaje, sino que ahora es él, son los grupos los protagonistas de dicho proceso.

Desde la perspectiva de la maestra, el ABP ha permitido que el alumnado vaya adquiriendo autonomía en su proceso de aprendizaje al tener que organizar sus trabajos y preparar 
las exposiciones como muestra del producto final. Es la motivación con la que ha trabajado este la que destaca: "Son esponjas y aprender a la primera cuando algo les motiva" (Reflexión de la maestra), aspecto que busca la maestra con la innovación.

El ABP ha hecho también que el alumnado tenga otra mirada hacia la maestra, también por lo propios cambios, según este, que esta ha introducido en su práctica docente, con los que ambos (maestra-alumnado) han mejorado y se han beneficiado

Alumna 1: a mi me ha gustado porque la seño incluso todos nosotros hemos aprendido a hacer cosas nuevas, porque por ejemplo la seño desde que se ha apuntado a este curso ha aprendido cosas nuevas como cuando mi madre estudia a lo mejor esas cosas no las había dado antes porque ella ha aprendido lo mismo que nosotros. (Entrevista alumnado)

La docente expresa cómo ha vivido la figura de la asesoría durante el proceso, viendo como una pieza la clave la necesidad del acompañamiento en los cambios en los procesos de enseñanza-aprendizaje, más concretamente en sus primeros pasos en la puesta en práctica del ABP en su aula

El saber que estabas ahí por si yo no lo enfocaba como debía pues me hizo que me relajara un poco. Porque ya te digo yo que sola jamás me hubiera metido en esto. (Entrevista maestra)

Al ser la asesoría una guía constante, una persona de referencia donde poder acudir y saber que va a encontrar respuestas a las dudas y posibilidades de hacer ante diferentes situaciones, le da seguridad y alejando el sentimiento de soledad ante el cambio, eliminando la incertidumbre con su presencia en cada uno de los momentos del proceso que requiere el trabajo por proyectos.

Por último, la asesoría en proceso ha favorecido la confianza en sí misma, descartando un rechazo a seguir trabajando en un futuro el ABP

Creo que he aprendido muchísimo en estos meses, y ahora sí me veo el curso que viene trabajando con mis futuros alumnos un proyecto de trabajo. Seguramente no me salga perfecto, pero confío en mí y se que algún día llegará. (Reflexión final de la maestra)

En cuanto a la influencia del centro educativo en la puesta en práctica del ABP en el aula por parte de la docente, el centro educativo tiene un papel fundamental para canalizar inquietudes de los maestros y maestras y potenciar la formación permanente de profesorado; así como las asesorías de los CEPs, recogiendo directamente de los docentes sus necesidades y ofreciéndoles información directa y orientación. Establecer este triángulo posibilitaría una mayor formación permanente, a partir de las necesidades e inquietudes detectadas y manifestadas en relación con las líneas educativas del centro, potenciando el desarrollo profesional docente a partir del cambio en las propias prácticas educativas

Una persona que se acerque a esa gente que está un poco desorientada y que le diga ¿a ti que te interesa? ¿qué inquietudes tienes? ¿En que te has formado ya? ¿qué te queda? Pues ya sabes que aquí existen cursos de esto y de lo otro, y aquí y allí. Creo que esto implicaría un poquito más al profesorado (Entrevista maestra, p. 9)

Si bien para dicho desarrollo profesional docente a partir del cambio educativo es necesario, además, cambiar las condiciones laborales, fundamentalmente las referidas al tiempo del que disponen maestros y maestras para la formación, de manera que esta se vea como algo necesario 
y parte integrante de la práctica educativa y no algo condicionado a la disponibilidad o no de tiempo.

Esta situación se solventaría, en cierta medida, con la formación en centros y el asesoramiento durante el proceso. No obstante, para potenciar ésta, así como la continuidad del cambio, sería necesario formar equipos de trabajo, por ciclos, para contrastar, discutir, proponer y eliminar el sentimiento de soledad ante el cambio educativo, además de poder dar continuidad a este, pudiéndose desarrollar un asesoramiento grupal durante el proceso.

\section{Conclusiones y discusión}

A lo largo de este estudio se ha podido observar como la docente ha vivido con gran aceptación la figura de la asesoría colaborativa durante el proceso de la puesta en práctica del ABP en su aula, ya que le ha ayudado a superar los primeros miedos que presentaba a la hora de llevarlo a cabo, tomando confianza para su posterior puesta en práctica en su aula y destacando su imprescindible presencia para poder introducir cambios en su quehacer docente, gracias a asesoramiento teórico-práctico realizado durante el proceso, facilitando una formación más experiencial y contextualizada.

Tal y como señalan Hargreaves y Fullan (2014), el "capital profesional”, una vez que empieza, actúa como mecanismo de arranque que impulsa el cambio de prácticas educativas. El trabajo colaborativo y la interacción entre docentes, y en este caso en concreto con la asesoría de formación, influye positivamente en los procesos de cambio, sintiendo un apoyo para avanzar en el desarrollo profesional. Desde aquí, también la docente señala que la figura de la asesoría durante el proceso le ha ayudado, destacando la necesidad del acompañamiento en los cambios del proceso de enseñanza-aprendizaje, sobre todo en sus primeros pasos en la puesta en práctica del ABP en su aula, ya que dicho acompañamiento ha favorecido reducir el sentimiento de soledad de la maestra en los procesos de cambio y la incertidumbre ante este, viviendo sus errores no como algo negativo sino como fuente para su aprendizaje, potenciado el contraste de ideas, el pensamiento y la reflexión colectiva.

Los procesos de innovación y cambio se enriquecen con procesos colaborativos y de intercambio docente y el poder vivir estos procesos acompañados de la figura de la asesoría colaborativa lo enriquecen aún más. La docente destaca que el poder conocer a esta figura, poder hablar con ella, tener una relación más cercana, implicaría al profesorado más en la formación.

Se ha podido comprobar que la reflexión y acompañamiento durante la puesta en práctica del ABP influye de manera directa en las creencias educativas del profesorado, viendo reflejado como la docente tiene una serie de creencias sobre diferentes situaciones que afectan a sus acciones y comportamientos, observándose en las actitudes que presenta para afrontarlas. Está claro que las creencias guían nuestras prácticas presentes, limitando o potenciando las acciones. La docente cree en la idoneidad del Aprendizaje Basado en Proyectos como medio que favorece la motivación e implicación del alumnado en su propio proceso de aprendizaje, pero esta creencia se ve enfrentada con otras creencias en cuanto a que el aprendizaje es un proceso individual, a la necesidad de tener diversos recursos para poder llevarlo a cabo y la falta de tiempo que ella expone.

Fullan y Hargreaves (2014) señalan que el mejor lugar para empezar un cambio es con uno mismo. Sus propias experiencias, frustraciones, ideales y sentido del yo son puntos de partida 
cruciales. Es por ello que durante el proceso de realización del proyecto en el aula la docente ha ido transformando su proceso de enseñanza experimentando diferentes emociones que han incidido en este cambio, reparando más en las posibilidades que en las dificultades del proceso de cambio de su acción innovadora.

Existen muchos elementos que dificultan el cambio metodológico y la innovación docente, y que hacen proliferar las prácticas rutinarias en el aula, como son la falta de recursos y de tiempo tanto para la preparación de las clases como para la realización de formación propia. Estas continuas quejas o resistencias al cambio, también llamadas malestar docente, son premisas que tiene continuamente la docente, instaurada en prácticas tradicionales, y que la frenan en procesos de innovación.

Pero el estar comprometida y el querer incorporar en el aula prácticas innovadoras hace ver que, tras esa "fachada tradicional", se esconde una docente con ganas de introducir cambios en su práctica y que va haciéndose consciente de la necesidad de este proceso de aprendizaje tanto para ella como para el alumnado.

Durante y al final del proceso, la docente va siendo más consciente de la necesidad del cambio metodológico en su aula, para que el alumnado se sienta protagonista de su propio aprendizaje, coincidiendo con otras investigaciones como las de García-Valcárcel y Basilotta (2017) y Barba-Martín, Sonlleva y García-Martín (2018), encontrándose más motivado y con mayores habilidades para el trabajo en grupo. En cuanto al trabajo en grupo el alumnado se hace consciente de que son capaces de lograr mucho más, desbloqueando el potencial individual y fomentando la creatividad (Johnson y Johnson, 2014). La maestra comienza a valorar esta modalidad de trabajo al observar que favorece en el alumnado el pensar e imaginar, la autonomía, la responsabilidad y autorregulación en su aprendizaje (Kaldi, Filippatou, Govaris, 2011), el trabajo colaborativo, la autoevaluación y coevaluación de su proceso, eliminando, así, sus prejuicios respecto al trabajo en grupo que se traducían distracciones y escaso trabajo. Este cambio en las creencias de la maestra provoca otro en su papel en el aula, pasa de transmisora del conocimiento y controladora de todos los aspectos del proceso de enseñanza-aprendizaje a ser guía y mediadora de estos.

En esta situación sería necesario abrir el debate sobre cual debería ser el papel a desempeñar en los centros educativos desde la perspectiva de asesoría en procesos para fomentar el desarrollo profesional de los docentes, requiriendo en ellos una actitud de aprendizaje basada en la reflexión sobre la propia práctica y desde el contexto en que esta se produce (Imbernon, 2001, 2007), sea cual sea la transformación metodológica a incorporar en sus aulas. Atendiendo a la experiencia de la maestra en su proceso de innovación sería necesario, para potenciar el cambio educativo y la continuidad de este, formar equipos de trabajo, por ciclos, para contrastar, discutir, proponer y eliminar el sentimiento de soledad ante, por lo que se podría contemplar el desarrollo de un asesoramiento grupal durante el proceso.

\section{Referencias}

Barba-Marín, R., Sonlleva, M. y García-Marín, N. (2018). "Presencia participación y progreso": el aprendizaje basado en proyectos en la trayectoria de una maestra en formación. Revista Electrónica Interuniversitaria de Formación del Profesorado, 21 (2), 13-25. 
Booth, T. y Ainscow, M. (2015). Guía para la educación Inclusiva: Desarrollando el aprendizaje y la participación en los centros escolares. Madrid. España: Fuhem.

Decreto 110/2003, de 22 de abril, por el que se regula el Sistema Andaluz de Formación Permanente del Profesorado. BOJA núm. 78. Sevilla, 25 de abril de 2003.

Decreto 93/2013 de 27 de agosto, por el que se regula la formación inicial y permanente del profesorado en la Comunidad Autónoma de Andalucía, así como el Sistema Andaluz de Formación Permanente del Profesorado. BOJA núm. 170, Sevilla 30 de agosto de 2013.

Flick, U. (2004). Introducción a la investigación cualitativa. Madrid. España: Morata.

Fullan, M. (2002). Los nuevos significados del cambio en educación. Barcelona. España: Octaedro. García-Valcárcel, A. \& Basilotta, V. (2017). Aprendizaje Basado en Proyectos (ABP): evaluación desde la perspectiva de alumnos de Educación Primaria. Revista de Investigación Educativa, 35 (1), 113-131.

Grossman, P.; Dean, Ch.; Kavanagh, S. y Herrmann, Z. (2019). Preparing Teachers for ProjectBased Teaching. Phi Delta Kappan, 100 (7), 43-48.

Hargreaves, A. y Fullan, M. (2014). Capital profesional. Madrid. España: Morata.

Hernández, F. (1996). Para comprender mejor la realidad. Cuadernos de Pedagogía, 243, 48-53. Hernández, F. y Ventura, M. (2008). La organización del currículum por proyectos de trabajo. Barcelona. España: Octaedro.

Imbernon, F. (2001). Claves para una nueva formación del profesorado. Investigación en el Aula, 43, 57-66.

Imbernon, F. (2007). Asesorar o Dirigir. El papel del asesor/a colaborativo en una formación permanente centrada en el profesorado y en el contexto. Revista electrónica Iberoamericana sobre Calidad, Eficacia y Cambio en Educación, 5 (1), 145-152.

Imbernon, F. (2013). 10 Ideas clave. La formación permanente del profesorado. Barcelona. España: Graó.

Imbernon, F. (2014). Calidad de la enseñanza y formación del profesorado. Barcelona. España: Octaedro.

Imbernon, F. (2017). Ser docente en una sociedad compleja. La difícil tarea de enseñar. Barcelona. España: Graó.

Jacobsen, M. (2019). A Multi-case Study of a Problem-based Learning Approach to Teacher Professional Development. (Doctoral thesis). Pepperdine University: ProQuest Dissertations Publishing.

Johnson, D.W. y Johnson, R.T. (2014). La evaluación del aprendizaje cooperativo. Como mejorar la evaluación individual a través del grupo. Madrid. España: S.M.

Kaldi, S, Filippatou, D. y Govaris, C. (2011). Project-Based Learning in Primary Schools: Efeccts on Pupils' Leaning and Attitudes. Education 3-13, 39 (1), 35-47.

Kilpatrick, W.H. (1918). The Proyect Method. Teachers College Record, 19, 319-335.

Martín, A. \& Rodríguez, S. (2015). Motivación en alumnos de Primaria en aulas con metodología basada en proyectos. Revista de Estudios e Investigación en Psicología y Educación, 0 (01), 058-062.

Mckinsey \& Company (2012). Educación en España. Motivos para la esperanza. Recuperado de https://www.mckinsey.com/featured-insights/europe/education-in-spain-reasons-for-hope/es-es 
ORDEN de 20 de mayo de 1992, por la que se regula el funcionamiento de los Centros de Profesores de la Comunidad Autónoma de Andalucía. BOJA núm. 68, Sevilla 18 de julio de 1992.

Pinya, C. y Roselló, M. (2014). El asesor de formación permanente del profesorado: perfil, trayectoria y funciones. Revista Complutense de Educación, 23 (3), 661-678.

Rodríguez, C. e Imbernon, F. (2016). El necesario cambio de la profesión docente en el contexto actual. Recuperado de http://eldiariodelaeducacion.com/2016/10/03/necesario-cambio-laprofesion-docente-contexto-actual/

Sainz, F. (1934). El método de proyectos en las escuelas rurales. Madrid. España: Revista Pedagogía.

Simons, H. (2011). El estudio de caso: teoría y práctica. Madrid. España: Morata.

Stake, R. (1998). Investigación con estudio de casos. Madrid. España: Morata.

Torrego, L. y Martínez, S. (2018). Sentido del método de proyectos en una maestra militante en los Movimientos de Renovación Pedagógica. Revista Electrónica Interuniversitaria de Formación del Profesorado, 21(2), 1-12.

Trujillo, F. (2012). Enseñanza basada en proyectos: una propuesta eficaz para el aprendizaje y el desarrollo de las competencias básicas. Eufonía-Didáctica de la Educación Musical, 55, 7-15.

Vázquez, R. y Angulo, F. (2003). Introducción a los estudios de casos. Málaga. España: Aljibe.

Ventura, M. (2008). Asesor es acompañar. Currículum y Formación del Profesorado, 12 (1), $1-14$.

Vergara, J. (2015). Aprendo porque quiero. Madrid. España: SM.

Wood, P. (1987). La escuela por dentro. La etnografía en la investigación cualitativa. Barcelona. España: Paidós.

Zeichner, K. (1995). Los profesores como profesionales reflexivos y la democratización de la reforma escolar. En AA.VV. Volver a pensar la educación (vol. III) (pp. 385-398). Madrid. España: Morata. 